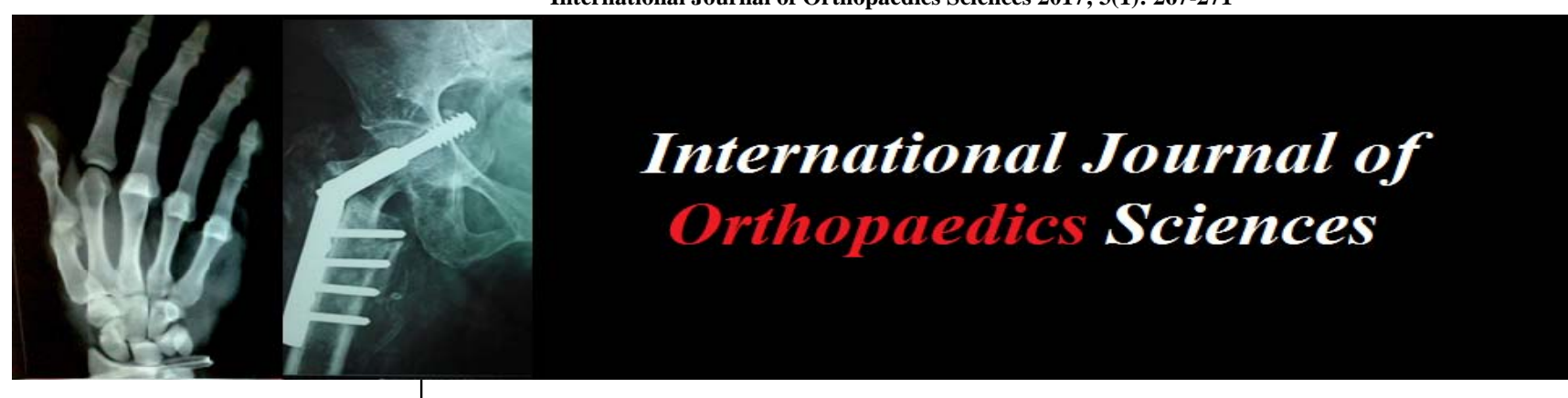

ISSN: 2395-1958

IJOS 2017; 3(1): 267-271

(C) 2017 IJOS

www.orthopaper.com

Received: 11-11-2016

Accepted: 12-12-2016

Dr. Sachin Katti

Senior Resident D'Ortho,

(DNB), Sanjay Gandhi Institute

of Trauma and Orthopaedics,

Bengaluru Karnataka, India

Dr. Madan Ballal

M S Ortho. Associate Professor \&

Chief, Sports Injury Centre,

Sanjay Gandhi Institute of

Trauma \& Orthopaedics,

Bengaluru Karnataka, India

Dr. HS Chandrashekar D Ortho, M S Ortho. Professor \& HOD, Sanjay Gandhi Institute of Trauma \& Orthopaedics,

Bengaluru Karnataka, India

Dr. Hemanth Kumar A M S Ortho. Professor \& HOD, Shoulder \& Arthroscopic

Surgery. B. M. Jain Hospital, Bengaluru Karnataka, India

Correspondence

Dr. Sachin Katti

Senior Resident D'Ortho,

(DNB), Sanjay Gandhi Institute

of Trauma and Orthopaedics,

Bengaluru Karnataka, India

\section{Functional outcome of arthroscopic Bankart repair for anterior shoulder instability}

\author{
Dr. Sachin Katti, Dr. Madan Ballal, Dr. HS Chandrashekar D and \\ Dr. Hemanth Kumar A
}

DOI: http://dx.doi.org/10.22271/ortho.2017.v3.i1e.41

Abstract

A prospective study was done on 21 patients with mean age of years (range yrs) underwent arthroscopic Bankart repair with suture anchors for anterior shoulder instability at Sports Injury Centre, Sanjay Gandhi Institute of Trauma and Orthopaedics, Bangalore. The study was conducted from 2014 to 2016 with a minimum follow-up of 1 year. Functional outcome was assessed with two outcome measures Walch-Duplays and UCLA scoring system. Range of motion, recurrence rate and patient satisfaction were evaluated. Functional outcome was good to excellent in most of patients, 2 patients $(9.5 \%)$ had fair results and one patient $(4.7 \%)$ had poor scoring. Most of patients showed improvement in range of motion with one patient didn't show improvement. Rate of recurrence was $4.7 \%$ ( 1 patient out of 21 ). In Conclusion, Arthroscopic Bankart repair for anterior shoulder instability provides good to excellent functional outcome, improved range of motions and low recurrence rate.

Keywords: Bankart repair, anterior shoulder instability, arthroscopic repair, suture anchors

\section{Introduction}

Shoulder joint gives wide range of motion at various positions in three-dimensional space by using the glenohumeral joint as a fulcrum. Shoulder is one of the most unstable and frequently dislocated joint in the body, accounting for more than $50 \%$ of all dislocations, with a $2 \%$ incidence in the general population ${ }^{[1]}$.

Main complication of shoulder dislocation is recurrent instability and it accounts for an average of $70-90 \%$ recurrence rate in patients between $20-40$ years of age. Following dislocation injuries occur to surrounding structure such as bone, cartilage and soft tissues, resulting in various pathological conditions which is less understood. During shoulder dislocations, humeral head is forced anteriorly out of glenoid cavity resulting in detaching fibrocartilaginous labrum from the anterior rim of glenoid cavity. This detachment of glenoid labrum is called Bankart lesion. Bankarts lesion is the most common and essential lesion in treatment of anterior shoulder instability.

Management is by reattachment of labro-ligamentous structure to the glenoid. Glenoid labrum plays important role in maintaining stability of glenohumeral joint ${ }^{[2]}$. Glenoid labrum deepens the socket and act as anchor point for ligaments and capsule.

Bankart treated anterior shoulder instability and Bankart lesion by open repair, this was published by Dickson and Devas in $1957^{[3]}$. Anterior shoulder instability can be treated either by open procedure or arthroscopic method. Since the description various methods of open and arthroscopic techniques have come to address various pathologies of shoulder instability.

Arthroscopic Bankarts repair has become the standard of care recently with advantages over open repair. In comparison to arthroscopic repair, open repair results in wide dissection, loss of external rotation, subscapularis tendon injury and post-operative pain ${ }^{[4,5]}$.

Recent advancements in the techniques, suture material, bioabsorbable anchors and concomitant treatment of other pathologies, arthroscopic repair has become a standard care ${ }^{[6]}$. The following study aim is to evaluate the functional outcome, range of movements and recurrence rate of arthroscopic Bankarts repair in anterior shoulder instability using a bioabsorbable suture anchors. 


\section{Materials and Methods}

The study was conducted at Sports Injury Centre, Sanjay Gandhi institute of trauma and Orthopaedics from 2014-2016. A total of 21 patients with recurrent anterior shoulder instability underwent arthroscopic Bankart repair.

A prospective study was conducted on patients with anterior shoulder instability aged between 15 to 50 years. Inclusion criteria included patients with recurrent anterior shoulder dislocation or subluxation, MRI showing Bankart lesion or diagnostic arthroscopy showing lesion. Exclusion criteria included patients with posterior instability, multidirectional instability, habitual dislocators, engaging Hill-Sach's lesion, bony Bankart or Hill-Sachs lesion with more than $25 \%$ bone loss and concomitant pathologies such as rotator cuff tear, SLAP lesion. Patients with failed arthroscopic or open repair and those who are medically unfit for surgery were excluded from the study.

Patients with anterior shoulder instability were evaluated clinically and all necessary information such as mode of injury, daily activities /sports, number of dislocations and range of movements were collected. Most of the patient had apprehension test positive. Radiographs in anterior-posterior, lateral, axillary and scapular Y view were taken. Following clinical and radiological examination MRI was done.

Informed consent was taken after explaining about the procedure, complications and intense rehabilitation protocol. Routine investigations were done and medical fitness was taken. All patients underwent arthroscopic Bankarts repair and intro-operative surgical details were noted down. Intense post-operative rehabilitation were done at weekly interval for 2 months and later on follow-up evaluation were done at 3 months, 6 months and 1 year.

Follow-up included subjective evaluation of pain, return to sports activity and limitation of work etc. Physical examination included range of movements, instability testing including apprehension test. Rate of recurrence were noted. Two outcome scoring measurements were used during the study Walch-Duplay and UCLA scoring systems.

Walch -Duplay Scoring system ${ }^{[7-9]}$ asses the sport / daily activity, stability, pain and mobility of patients. A total of 100 score is given and each parameter has maximum of 25 points. Score between 90-100 is considered excellent, 75-89 good, 5074 as average and less than 50 as poor. Patient were followed with this scoring system preoperatively and at regular interval of 3 months, 6 months and at 1 year post-operatively.

UCLA (University of California Los Angles) Scoring system ${ }^{[10]}$ was used for the functional outcome at 1 year of follow-up. The UCLA scoring system consists of 5 parameters namely Pain, Function, Active forward flexion, Strength and patient satisfaction. The maximum score is 35 . a score between 32-35 is excellent, 29-31 is good, 21-28- is average and less than 20 as poor.

Functional outcome were evaluated with Walch-Duplay score at 3 months, 6 months and at 1 year and UCLA score were done at 1 year of follow-up. Range of movements and complications were assessed. Statistical analysis was done comparing preoperative and postoperative scores, range of movements and other variables.

Recurrence of shoulder dislocation, persistent instability, failure to return to full activity and need for another procedure/ surgery was considered as treatment failure.

\subsection{Operative Technique}

All the operations were done by a senior surgeon and performed using standard technique. General anesthesia is preferred because better patient positioning. General anesthesia is given with the endotracheal tube taped securely on the site opposite to the side of surgery.

Pre-operatively second generation cephalosporin antibiotic is administered.

Patient is positioned in lateral decubitus position with arm in 40-45 degrees of abduction and 10-15 degrees of forward flexion and neutral rotation using adhesive traction. Bony prominences were well padded. Skin preparation, painting draping and surface markings was done.

Superficial skin markings of bony anatomical landmarks is done to guide portal placement. Standard Posterior portal is the primary viewing portal and is placed in the posterior soft spot in a standard manner. It is usually placed $2 \mathrm{~cm}$ inferior and in line with posterolateral aspect of the acromion.

Anteriosuperior portal was established by outside in technique, with a $18 \mathrm{G}$ spinal needle inserted at $1 \mathrm{~cm}$ anterior to the acromion and $2 \mathrm{~cm}$ lateral to coracoid process. Anterior midglenoid portal was established just over the superior border of subscapularis tendon.

Arthroscopic examination is done through anterior and posterior portals, assessment of glenoid labrum, capsuloligamentous structures, articular surfaces, Hill-Scah's lesion and any bone loss is evaluated. Anterior stabilization of Bankarts lesion is done using minimum of 2 double loaded suture anchors. Glenoid is evaluated for labral pathology and the bone loss is quantified. The labrum is completely freed from the glenoid neck and allowed to float freely up to the level of the glenoid face. The glenoid bone at the neck is prepared by removing any fibrous tissue. Shaver can be used to lightly 'dust' the neck to reveal raw bone but care is taken to avoid removing any bone stock.

The first pilot hole for the inferior most anchor is created by inserting a $2 \mathrm{~mm}$ drill bit with a self stopper, on the face of articular cartilage of the glenoid around the 5-o'clock position. It is ensured that the suture anchor is placed below the subchondral bone (2-3mm below).

A suture retriever is passed under the Bankart lesion. Through antero-superior portal the suture near the labrum is brought out through the labrum and then midglenoid portal in a retrograde fashion. This suture limb is designated as "Post" and to ensure that the knot remains on the capsular side of glenoid outside the articular surface. This results in anatomical restoration of labrum height and depth. Suture are tied using a Duncan loop sliding locking knot and secured with multiple reverse half hitches.

Second hole is drilled at 3.30 to 4.30 O'clock positions and accessory anchors are used based upon extent and size of labral tissue. If anteroinferior capsular laxity is treated by plication of redundant capsule. A final evaluation of the joint done from the anterior superior portal and documented which shows the humeral head is balanced in the centre of the glenoid.

Postoperatively the shoulder is immobilized for 4 weeks. For the four weeks patient is advised to do pendulum exercises. After 4 weeks main emphasis is given on regaining flexion range of motion, Scapular stabilization and isometric rotator cuff exercises are started. From 8th week onwards muscle strengthening exercises, mobilization exercises and proprioception are taught. Recreational sports and noncontact sports were allowed after 4 months and contact sports after 6months.

\section{Results and Analysis}

A total of 21 patients were followed up for 1 year. 19 patients 
were male and 2 female patients. The mean age at surgery was 27.19(range 17-44) years. The mean number of shoulder dislocations before surgery was $3.10 \pm 1$. 4. Most of the patient $66.7 \%{ }^{[14]}$ were in recreational sports, $14.3 \%{ }^{[3]}$ were doing daily activities of life and only $19 \%{ }^{[4]}$ were involved in competitive sports. Most of the patients had good to excellent outcomes in terms of Walch-Duplay score and the score from $60 \pm 9$ preoperatively improved to $90 \pm 14$ postoperatively $(p \leq 0.05)$.

UCLA score at 1 year of follow-up showed excellent to good results in $85.7 \%{ }^{[17]}$ patients and $9.5 \%$ (2) fair results. $1(4.7 \%)$ patient had poor functional outcome. One patient $(4.7 \%)$ had recurrence rate and persistent instability. Improvement in range of motions was seen in all patients postoperatively. The mean and SD of external rotation improved from $74 \pm 8$ to $90 \pm 7$ while the forward flexion improved from $141 \pm 12$ to $159 \pm 5$ and Internal rotation improved from $54 \pm 7$ to $67 \pm 4$. Apart from breakage of bio-anchor in two patient, there were no intraoperative complications. One patient had supraspinatus tear at 8th month of follow-up with limitation of abduction and external rotation. $90.5 \%$ (19 patients out of 21$)$ of the patients were satisfied with UCLA rating. Recurrence rate or the failure rate was $4.7 \%$ ( 1 in 21 patients).

\section{Discussion}

With recent enthusiasm for recreational and sporting activities, the incidence of Shoulder instability is on rise, especially in young athletics \& active population. Anterior instability is most common type of shoulder instability seen and trauma is the main cause for it.

Glenoid labrum forms an important stabilizer of shoulder joint. It is fibrous rim around the glenoid fossa, which is triangular in cross section. It deepens glenoid fossa by $50 \%$ and increases humeral contact to $75 \%{ }^{[2,11,12]}$.

Bankart lesion, detachment of the antero-inferior labrum causes recurrent anterior instability ${ }^{[13]}$. The Bankart repair as described by Rowe et al., ${ }^{[14]}$ involves suturing the avulsed capsulolabral complex to the anterior glenoid rim. Anterior shoulder instability can be treated either by open procedure or arthroscopic method ${ }^{[4,5]}$

Bankart treated anterior shoulder instability and Bankart lesion by open repair, this was published by Dickson and Devas in $1957^{[3]}$.

In 1993, Detrisac ${ }^{[15]}$ first reported arthroscopic stabilization using metal staple with high recurrence rate and high risk of implant failure. Since then lot of advances have taken place in terms of technique, suture material, bioabsorbable anchors and concomitant treatment of other pathologies, arthroscopic repair has become a standard care ${ }^{[6]}$.

Arthroscopic method has various advantages when compared to open procedure. It offers minimally invasive approach with cosmetic acceptability and less surgical time, trauma and blood $[4,5]$. Arthroscopic procedure can be done as a day care procedure. Postoperative pain is less, recovery and rehabilitation is faster. Open procedure results in subscapularis tendon disruption and postoperative tendon rupture have been reported ${ }^{[16]}$. Limitation in range of motion, especially external rotation, is one of the major drawbacks in terms of functional outcome after open Bankart reconstruction. Various studies have shown good postoperative range of motions and with improvement in external rotation ${ }^{[17,18]}$ following arthroscopic repair.

Lot of advancement have been made in terms of suture anchor and suture material $[19,20]$ in terms of strength, biocompatibility, improved visualization and easier revision surgery. New sutures containing Ultra High Molecular Weight Polyethylene have become gold standard. Bioabsorbable and biocompatible suture anchor have come to minimize complications during revision, diminished concern regarding migration. In this study we most commonly used two to three anchors based on the size of Bankart lesion. Better suture anchor insertion techniques ${ }^{[22]}$, care and knot securing may decrease complications of screw breakage, pull out etc.

Bankart repair was done using two to three suture anchors based upon the bankart lesion size. Anteroinferior capsuloligamentous laxity was treated with capsular placation there by augmenting the Bankarts repair ${ }^{[21]}$. Capsular laxity may affect the outcome of surgery ${ }^{[4,17,21]}$.

Most of the patients were young with mean age of 27.19 years and involved in recreational sports and daily activities of life. Most of the patient returned to pre-injures status and $90.5 \%$ of the patient were satisfied with the treatment outcome. $90.5 \%$ of the patients had good range of motion and there was no external rotation restriction. With Walch-Duplay scoring $(90 \pm 14)$ and UCLA scoring system $(85.7 \%)$ most of the patients showed good to excellent results in terms of functional outcomes. Various studies such as Cole BJ et al. [4], Mishra DK et al. ${ }^{[21]}$, and IdE J et al. ${ }^{[24]}$ have shown similar good to excellent functional results following arthroscopic bankart repair. In this study we had $4.7 \%$ of failure rate which is similar to other studies. Gartsman et al. ${ }^{[17]}$ had a failure rate of $7.5 \%$, Mishra et al. ${ }^{[21]}$ had $7 \%$ and Amit ${ }^{[18]}$ et al. had $7 \%$ of failure rate.

Limitation of this study is short term follow-up and small study group. Presence of concomitant pathologies along with bankart lesion may affect the functional outcome in shoulder instability repair, need to be explored.

In conclusion, arthroscopic bankart repair gives good to excellent functional and clinical outcome in anterior shoulder instability.

Table 1: Range of Movements

\begin{tabular}{|c|c|c|c|c|c|}
\hline Mean \pm SD & FE & CBA & ER1 & ER2 & IR \\
\hline Pre op & $141 \pm 12$ & $42 \pm 4$ & $59 \pm 6$ & $74 \pm 8$ & $54 \pm 7$ \\
\hline $3 \mathrm{~m}$ & $150 \pm 11$ & $49 \pm 3$ & $65 \pm 5$ & $77 \pm 7$ & $60 \pm 6$ \\
\hline $6 \mathrm{~m}$ & $158 \pm 6$ & $50 \pm 2$ & $68 \pm 4$ & $86 \pm 8$ & $67 \pm 5$ \\
\hline $12 \mathrm{~m}$ & $159 \pm 5$ & $50 \pm 2$ & $68 \pm 4$ & $90 \pm 7$ & $67 \pm 4$ \\
\hline P value & $p \leq 0.05$ & $p \leq 0.05$ & $p \leq 0.05$ & $p \leq 0.05$ & $p \leq 0.05$ \\
\hline
\end{tabular}

FE-Forward Elevation CBA-Cross Body Adduction

ER1-External rotation by side of body/neutral

IR1-Internal rotation by side of body/neutral

ER2- External rotation at $90^{\circ}$ Abduction

Table 2: UCLA Score at 1 year

\begin{tabular}{|c|c|c|}
\hline Ucla Shoulder Rating At 1 Year & \% \\
\hline Poor $<20$ & 1 & 4.761905 \\
\hline Fair (21-27) & 2 & 9.52381 \\
\hline Good (28-31) & 9 & 42.85714 \\
\hline Excellent (32-35) & 9 & 42.85714 \\
\hline
\end{tabular}

Table 3: Walch Duplay Score

\begin{tabular}{|c|c|c|c|c|c|}
\hline \multicolumn{5}{|c|}{ Walch Duplays Score } & \\
\hline Mean \pm SD & $\begin{array}{c}\text { Sport Or } \\
\text { Daily Activity }\end{array}$ & Stability & Pain & Mobility & Total \\
\hline Pre op & $14 \pm 2$ & $16 \pm 5$ & $16 \pm 3$ & $15 \pm 6$ & $60 \pm 9$ \\
\hline 3 months & $15 \pm 1$ & $24 \pm 3$ & $20 \pm 5$ & $16 \pm 5$ & $77 \pm 8$ \\
\hline 6months & $15 \pm 1$ & $24 \pm 3$ & $20 \pm 5$ & $16 \pm 5$ & $88 \pm 14$ \\
\hline At 1 year & $18 \pm 5$ & $24 \pm 3$ & $22 \pm 5$ & $24 \pm 5$ & $90 \pm 14$ \\
\hline P value & $p \leq 0.05$ & $p \leq 0.05$ & $p \leq 0.05$ & $p \leq 0.05$ & $p \leq 0.05$ \\
\hline
\end{tabular}




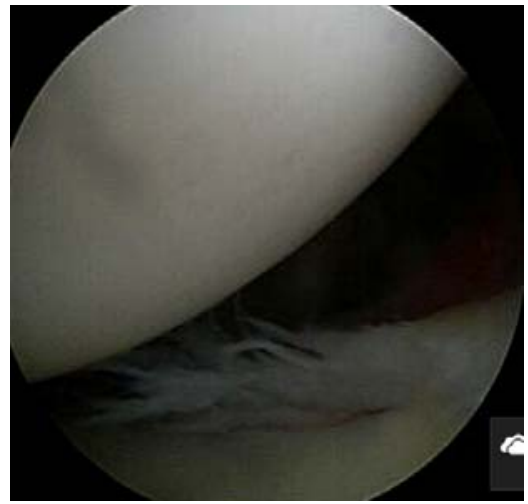

A. Bankart;s Lesion

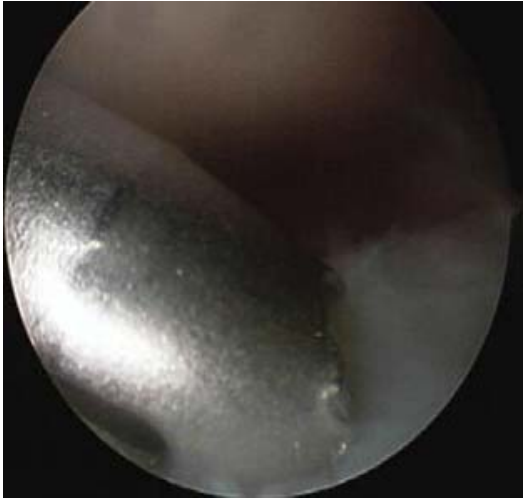

C. Drilling

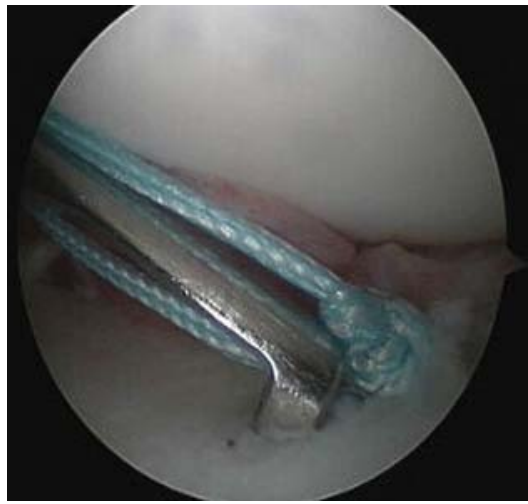

E. Anchor Knot tying

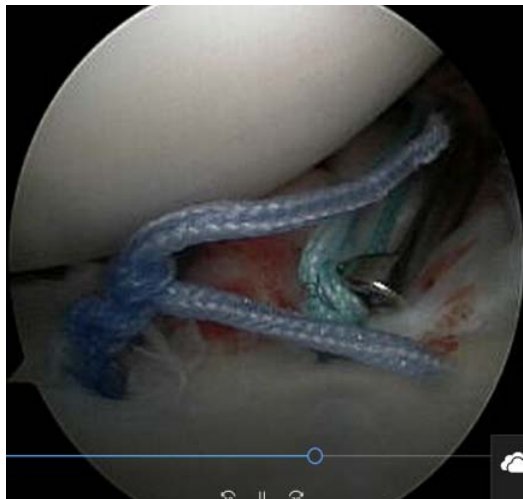

G. Suture retrival from 2nd Anchor

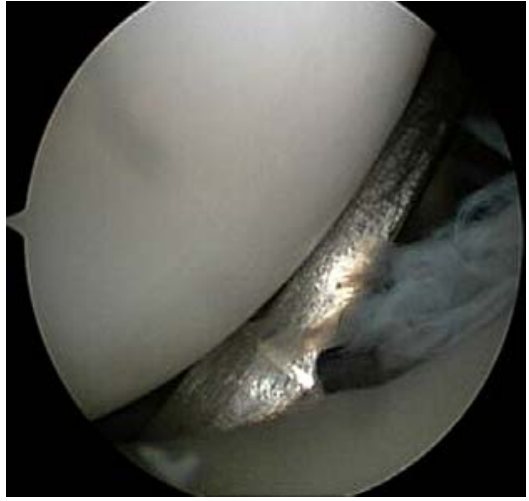

B. Elevating lesion

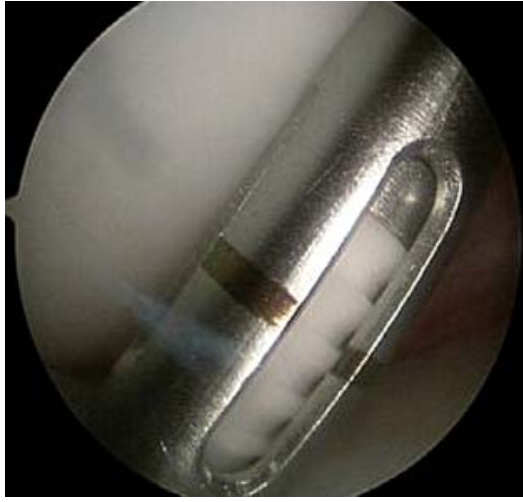

D. Anchor Insertion

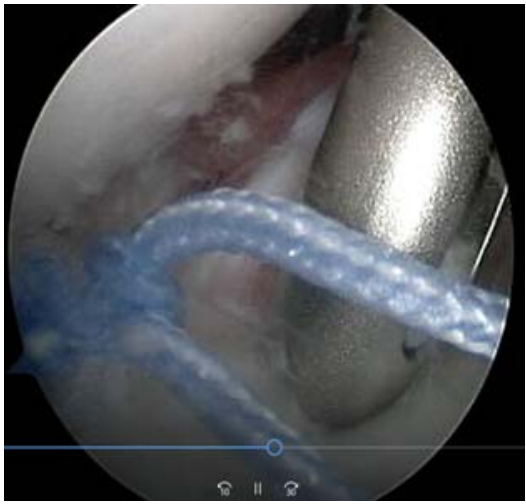

F. Second Anchor insertion

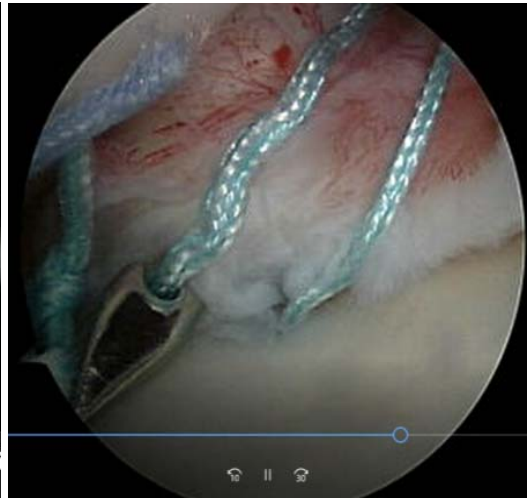

H. Bankarts Fixation with 2 Anchor

Fig 1: Bankarts Repair 


\section{References}

1. Hovelius L. Recurrences after initial dislocation of the shoulder. Results of prospective study of treatment. J Bone Joint Surg Am. 1983; 65(3):343-349.

2. Bigiliani LU, Newton PM, Steinmann SP. Glenoid rim lesions with recurrent anterior dislocation of the shoulder. Am J Sports Med. 1998; 26:41-45

3. Dickson JW, Devas MB. Bankart's operation for recurrent dislocation of shoulder. J Bone Joint Surg (Br), 1957; 39:114-119,

4. Cole BJ, Insalata L, Irrgang J. Comparison of arthroscopic and open anterior stabilization: a two to six year followup study. J Bone Joint Surg. 2000; 82A:1108-1114.

5. Fabbriciani C, Milano G, Demontis A, Fadda S, Ziranu F, Mulas PD. Arthroscopic versus open treatment of Bankart lesion of the shoulder: A prospective randomized study. Arthroscopy. 2004; 20:456-62.

6. Sperling JW, Smith AM, Cofield RH, Barnes S. Patient perceptions of open and arthroscopic shoulder surgery. Arthroscopy. 2007; 23:361-6.

7. Walch G. The Walch-Duplay Score for Instability of the Shoulder. Directions for the use of the quotation of anterior instabilities of the shoulder. Abstracts of the First Open Congress of the European. Society of Surgery of the Shoulder and Elbow, Paris, 1987, 51-55.

8. Amstutz HC, Sew Hoy AL, Clarke IC. UCLA anatomic total shoulder arthroplasty. Clin Orthop Relat Res. 1981; 155:7-20.

9. Khiami F, Sariali E, Rosenheim M, Hardy P. Anterior shoulder instability arthroscopic treated outcomes measures. The WOSI Correlates with the Walch-Duplay score. Orthopaedics \& Traumatology: Surgery \& Research 2012; 98:48-53.

10. Wright RW, Baumgarten KM. Shoulder outcomes measures. J Am Acad Orthop Surg. 2010; 18:436-444. (PubMed: 20595136)

11. Bigiliani LU, Newton PM, Steinmann SP. Glenoid rim lesions with recurrent anterior dislocation of the shoulder. Am J Sports Med. 1998; 26:41-45.

12. Sugaya H, Moriishi J, Dohi M. Glenoid rim morphology in recurrent anterior glenohumeral instability. J Bone Joint Surg. 2003; 85A:878-884.

13. Pappas AM, Goss TP, Kleinman PK. Symptomatic shoulder instability due to lesions of the glenoid labrum. Am J Sports Med. 1983; 11:279-88.

14. Rowe CR, Patel D, Southmayd WW. The Bankart procedure: a long term end-result study. J Bone Joint Surg. 1978; 60A:1-16.

15. Detrisac DA, Jhonson LL. Arthroscopic shoulder capsuloraphy using metal staples. Orthopaedic Clinics of North America 1993; 24:71-78.

16. Scheibel M, Tsynman A, Magosch P, Schroeder RJ, Habermeyer P. Postoperative subscapularis muscle insufficiency after primary and revision open shoulder stabilization. Am J Sports Med. 2006; 34:1586-93.

17. Gartsman GM, Roddey TS, Hammerman SM. Arthroscopic treatment of anterior-inferior glenohumeral instability. Two to five-year followup. J Bone Joint Surg Am. 2000; 82:991-1003.

18. Amit Mishra, Pulak Sharma, Deepak Chaudhary. Analysis of the functional results of arthroscopic Bankart repair in posttraumatic recurrent anterior dislocations of shoulder. Indian J Orthop. 2012; 46(6):668-674.

19. Barbar FA. Cyclic load testing and ultimate failure strength of bio-degradable glenoid anchors. Arthroscopy
2008; 24:224-228.

20. Wust DM. Mechanical and handling properties of braided polyblend polyetylene sutures in comparison to braided polyester and monofilament polydioxanone sutures. Arthroscopy. 2006; 22:1146-1153.

21. Mishra DK, Fanton GS. Two-years outcome of arthroscopic Bankart repair and electrothermal-assisted capsulorrhaphy for recurrent traumatic anterior shoulder instability. Arthroscopy. 2001; 17:844-9.

22. Kevin D Plancher, Stephanie CP. Arthroscopic Bankarts Repair with Suture Anchors: Tips for Success. Oper Tech Sports Med. 2013; 21:192-200.

23. Szyluk K. Outcomes of arthroscopic suture-anchor repair of isolated post-traumatic Bankart lesions : Med Sci Monit, 2015; 21:2331-2338

24. Ide J, Maeda S, Takagi K. Arthroscopic Bankart repair using suture anchors in athletes: patient selection and postoperative sports activity. Am J Sports Med. 2004; 32:1899-905. 